\title{
Robust stability of sampled-data systems under possibly unstable additive/multiplicative perturbations
}

\section{$\operatorname{AUTHOR}(S)$ :}

Hagiwara, T; Araki, M

\section{CITATION:}

Hagiwara, T ... [et al]. Robust stability of sampled-data systems under possibly unstable additive/multiplicative perturbations. IEEE Transactions on Automatic Control 1998, 43(9): 1340-1346

\section{ISSUE DATE:}

1998-09

URL:

http://hdl.handle.net/2433/39968

\section{RIGHT:}

(c) 1998 IEEE. Personal use of this material is permitted. However, permission to reprint/republish this material for advertising or promotional purposes or for creating new collective works for resale or redistribution to servers or lists, or to reuse any copyrighted component of this work in other works must be obtained from the IEEE. 


\title{
Robust Stability of Sampled-Data Systems Under Possibly Unstable Additive/Multiplicative Perturbations
}

\author{
Tomomichi Hagiwara and Mituhiko Araki
}

\begin{abstract}
This paper applies the FR-operator technique to the robust stability problem of sampled-data systems against additive/multiplicative perturbations, where a reasonable class of perturbations consists of unstable as well as stable ones. Assuming that the number of unstable modes of the plant does not change, we show that a small-gain condition in terms of the FR-operator representation (which is actually equivalent to a small-gain condition in terms of the $L_{2}$-induced norm) is still necessary and sufficient for the sampled-data system to be robustly stable against $\boldsymbol{h}$-periodic perturbations, in spite of their possible instability. The result is derived by a Nyquist-type of arguments. Next, a necessary and sufficient condition for robust stability against linear time-invariant (LTI) perturbations is also given. Furthermore, we show that if the plant is either single-input or single-output, the condition can be reduced to a readily testable form. Finally, we clarify when the small-gain condition becomes a particularly poor measure for robust stability.
\end{abstract}

Index Terms-FR-operator, impulse modulation formula, LTI perturbations, periodic perturbations, robust stability.

\section{INTRODUCTION}

The robust stability analysis and $H_{\infty}$ control of sampled-data systems were studied intensively over the past several years (e.g., [1]-[3], [6]-[10], [17], [19], [20], [23]-[26], and [28]-[30]). Many of these studies use the $L_{2}$-induced norm, and the small-gain theorem has been the typical tool. Recently, however, frequency-domain methods for sampled-data systems were developed, and their link to the $L_{2}$-induced norm method was established [2], [28]-[30] (see also [8], [9], [11], [13], [15], [17], and [24] for other related frequencydomain studies).

There are two main contributions included in this paper. The first is to have derived the necessary and sufficient conditions for robust stability of sampled-data systems under possibly unstable perturbations (it should be noted that we confine ourselves to additive/multiplicative perturbations in this paper; unstable perturbations naturally arise in these type of perturbations). When perturbations may be unstable, the small-gain theorem cannot be applied, and hence no robust stability conditions have been obtained. In this paper, under mild assumptions (including the assumption that the number of unstable modes of the plant does not change), necessary and sufficient conditions are derived through a Nyquist-type of argument with the FR-operator technique [2], making use of its frequency-domain nature. Section IV deals with the case of linear $h$-periodic perturbations and Section V the case of linear time-invariant (LTI) perturbations. It turns out that a smallgain condition in terms of the FR-operator representation (which is actually equivalent to one in terms of the $L_{2}$-induced norm) is still necessary and sufficient for the case of linear $h$-periodic perturbations, in spite of their possible instability; note that this result extends the one given in [25] and is a sampled-data counterpart to [5]. Similarly, the necessary and sufficient condition for LTI perturbations extends the one given in [9].

The second main contribution of this paper is to have clarified when the small-gain condition becomes an extremely poor measure

Manuscript received May 21, 1997.

The authors are with the Department of Electrical Engineering, Kyoto University, Yoshida, Sakyo-ku, Kyoto 606-01, Japan (e-mail: hagiwara@kuee.kyoto-u.ac.jp).

Publisher Item Identifier S 0018-9286(98)06117-0. 
for robust stability analysis and synthesis against LTI perturbations. The key for the success in obtaining this result is that we employed a unified method (i.e., Nyquist-type of argument with FR-operators) to deal with both linear $h$-periodic and LTI perturbations. The observation of the obtained result leads us to an insight into the design problem of a robustly stabilizing controller.

Although we use the FR-operator in this paper, it might be possible to derive the same results using an essentially equivalent notion (the frequency response operator defined via a lifting-based transfer function [30]). In the present authors' views, the advantages of adopting the FR-operator in the very context of the problem we study here seem to be as follows. First, the FR-operator is an operator on $l_{2}$, and as given in Section III-C, the notion of a determinant can easily be introduced to it in a quite similar way to the finite-dimensional case. Moreover, because of this, the interpolation problems that we need to solve for the necessity arguments of robust stability conditions reduce to intuitive and tractable ones (Appendix B).

Notation and Terms: $\|X\|$ denotes the maximum singular value of $X$ if $X$ is finite dimensional and the $l_{2}$-induced norm of $X$ if it is infinite dimensional. A system is denoted by a Roman/Greek capital letter, e.g., $P$ and $\Psi$, and its frequency-domain representation (including the $h$-periodic counterpart if it is $h$-periodic; see Section III-B) is denoted by putting a hat on it, e.g., $\hat{P}$ and $\hat{\Psi}$; a Roman letter is for a continuous-time system while a Greek letter is for a discrete-time system, in general. A mode of a continuous-time LTI system is an eigenvalue of its state transition matrix and is unstable if it lies in the closed right half-plane.

\section{Problem Formulation}

The purpose of this paper is to study robust stability of the sampled-data system $\Sigma_{s}$ shown in Fig. 1, which consists of the continuous-time plant $P$, the anti-aliasing filter $F$, and the discretetime compensator $\Psi$, together with the ideal sampler $\mathcal{S}$ and the (arbitrary but given generalized) hold $\mathcal{H}$, both operating at sampling period $h$ synchronously. The nominal plant is denoted by $P_{0}$, and the actual plant $P$ lies somewhere in a prescribed set $\mathcal{P}$ including $P_{0}$. In this paper, we consider the case where the set $\mathcal{P}$ is described with additive or multiplicative perturbations. Namely, we consider one of the following equations:

$$
\begin{aligned}
& P=P_{0}+W_{1} \Delta W_{2}, \quad P=P_{0}\left(I+W_{1} \Delta W_{2}\right) \\
& P=\left(I+W_{1} \Delta W_{2}\right) P_{0} .
\end{aligned}
$$

Here, $W_{1}$ and $W_{2}$ are appropriate known stable weights, and $\Delta$ denotes the perturbation, which lies in a prescribed set to be defined later.

It is generally true that we must consider an unstable (as well as stable) $\Delta$ unless we assume that the unstable part of the plant is perfectly known, which is a strong assumption (for example, if the location of an unstable mode is uncertain, we will be led to an unstable additive perturbation). This motivates us to study the robust stability problem under possibly unstable additive/multiplicative perturbations. The small-gain theorem cannot deal with this type of perturbation, and this paper aims at establishing a Nyquist-type approach to this problem. Throughout the paper, we make the following assumptions.

A1) $P_{0}$ is strictly causal and finite-dimensional LTI (FDLTI).

A2) $P$ is strictly causal, finite dimensional, and is LTI or linear $h$-periodic.

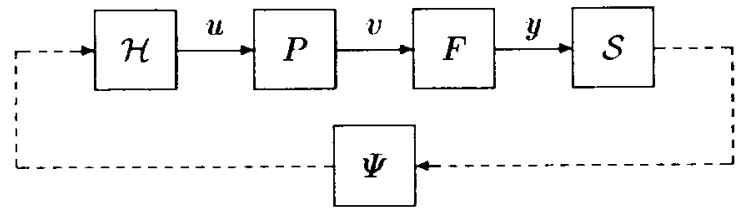

Fig. 1. Sampled-data system $\Sigma_{s}$.

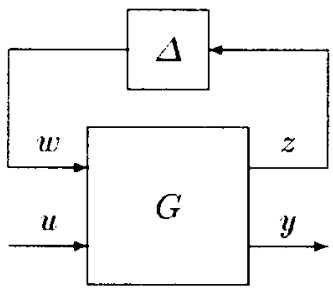

Fig. 2. Perturbed plant with an anti-aliasing filter.

A3) In (1), $W_{1}$ and $W_{2}$ are given stable causal FDLTI systems, and $\Delta$ is a causal linear $h$-periodic system with the " $L_{\infty}$ norm" of $\hat{\Delta}$ being less than one: $\|\hat{\Delta}\|_{\infty}<1$.

The definition of the $L_{\infty}$-norm of the frequency-domain representation $\hat{\Delta}$ corresponding to the linear $h$-periodic system $\Delta$ will be deferred to Section III-B.

By A2), it follows that the actual plant $P$ can be associated with a discrete-time state transition matrix over the interval $[0, h]$ (i.e., the monodromy matrix). Let us call the number of the eigenvalues of the monodromy matrix with magnitude no smaller than one, the number of the unstable modes of $P$. Now, our next assumptions are as follows [A5) is assumed for simplicity].

A4) $P$ has exactly as many unstable modes as $P_{0}$.

A5) $F$ is causal and FDLTI.

The mapping from $u$ to $y$ coincides with that of the system shown in Fig. 2, where $G$ is an appropriately formed FDLTI generalized plant. Note that

$$
G_{11}=0
$$

by (1). For this $G$, we assume the following [A6) is standard from [6], while A7) is for simplicity].

A6) The system $G_{21}$ from $w$ to $y$ is strictly causal.

A7) $G_{12}$ and $G_{21}$ have no modes on the imaginary axis.

The above assumptions are satisfied if $F$ is strictly causal and if $P_{0}$ and $F$ have no modes on the imaginary axis. Finally, we assume the following (most of the holds studied so far in the literature, including the zero-order hold, satisfy it).

A8) $\mathcal{H}$ is a finite-response $C_{1}$ hold [2].

Definition 1: The set of $\Delta$ satisfying Assumptions A2)-A4) is denoted by $\Delta$. The set of plants $P$ corresponding to $\Delta$ is denoted by $\mathcal{P}$.

Definition 2: The sampled-data system $\Sigma_{s}$ is robustly internally stable if $\Psi$ internally stabilizes any $P \in \mathcal{P}$ (in the sense of [6] and [21]). It is uniformly robustly internally stable if it is robustly internally stable and if $\inf _{P \in \mathcal{P}} \bar{\kappa}_{P}>0, \sup _{P \in \mathcal{P}} \underline{\kappa}_{P}<0$, where $\bar{\kappa}_{P}$ and $\underline{\kappa}_{P}$, respectively, denote the upper and lower gain margin in $\mathrm{dB}$ corresponding to the plant $P$.

Now, our robust stability problem is posed as follows. 
Problem 1: Assume A1)-A8). Given an LTI compensator $\Psi$ that internally stabilizes the nominal plant $P_{0}$, find a necessary and sufficient condition for uniform robust internal stability of the sampleddata system $\Sigma_{s}$.

\section{PRELIMINARIES}

\section{A. FR-Operator of Sampled-Data Systems}

Let us consider the nominal sampled-data system shown in Fig. 3, where $G$ is as introduced in the preceding section. It can be described by the FR-operator representation [2], [15] as

$$
\begin{gathered}
\underline{G}_{\Psi}(j \varphi)=\frac{1}{h} \frac{G_{12} H}{}(j \varphi) \cdot \hat{\Lambda}\left(e^{j \varphi h}\right) \cdot \underline{G_{21}}(j \varphi), \\
\varphi \in\left(-\omega_{s} / 2, \omega_{s} / 2\right]=: \mathcal{I}_{0}
\end{gathered}
$$

where $\omega_{s}:=2 \pi / h$ denotes the sampling angular frequency,

$$
\begin{aligned}
& \varphi_{m}:=\varphi+m \omega_{s} \quad(m=0, \pm 1, \pm 2, \cdots)
\end{aligned}
$$

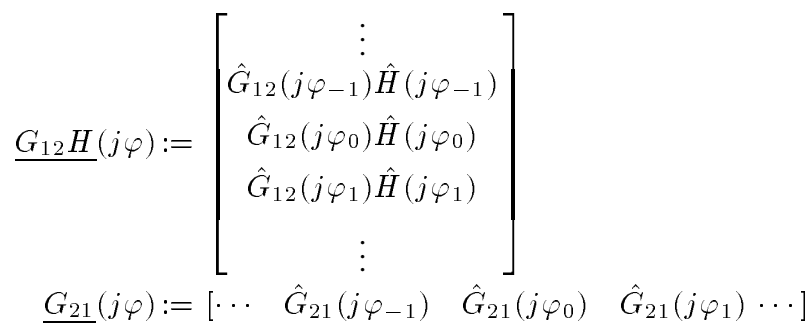

and $\hat{\Lambda}$ is given by

$$
\hat{\Lambda}:=\hat{\Psi}\left(I-\hat{\Pi}_{0} \hat{\Psi}\right)^{-1}, \quad \Pi_{0}:=\mathcal{S F} P_{0} \mathcal{H} .
$$

$\hat{H}(s)$ denotes the "transfer matrix" of the hold $\mathcal{H}$ (e.g., $\hat{H}(s)=$ $\left(1-e^{-s h}\right) I / s$ for the zero-order hold).

For each $\varphi \in \mathcal{I}_{0}, G_{\Psi}(j \varphi)$ defines a bounded operator on $l_{2}$. Let us denote the set of $\underline{G}_{\Psi}(j \varphi)\left(\varphi \in \mathcal{I}_{0}\right)$ by $\mathcal{G}_{\Psi}$, and define

$$
\left\|\mathcal{G}_{\Psi}\right\|_{\infty}:=\max _{\varphi \in \mathcal{I}_{0}}\left\|\underline{G}_{\Psi}(j \varphi)\right\|
$$

where the norm in the right-hand side denotes the induced norm on $l_{2}$. $\left\|\mathcal{G}_{\Psi}\right\|_{\infty}$ is called the $H_{\infty}$-norm of the FR-operator $\mathcal{G}_{\Psi}$ [2] and is known to coincide with the $L_{2}$-induced norm from $w$ to $z$ of the sampled-data system [2], [29].

An important property of (3) is that it defines a finite-rank operator on $l_{2}$.

\section{B. FR-Operator of h-Periodic Continuous-Time Systems}

Next, let us quickly review the study of [27] on the frequency response of an $h$-periodic continuous-time system and introduce the notion of the $L_{\infty}$-norm for $\hat{\Delta}$.

Without loss of generality (see [18] and [27]), let us employ the following "realization" of $\Delta$ :

$$
\frac{d x_{\Delta}}{d t}=A_{\Delta} x_{\Delta}+B_{\Delta}(t) u_{\Delta}, \quad y_{\Delta}=C_{\Delta}(t) x_{\Delta}+D_{\Delta}(t) u_{\Delta}
$$

where $A_{\Delta}$ is a constant matrix and $B_{\Delta}(t), C_{\Delta}(t)$, and $D_{\Delta}(t)$ are $h$-periodic matrices that are continuous and bounded on the interval $[0, h)$. In the following, it is enough to study the case where $A_{\Delta}$ has no eigenvalues on the imaginary axis (i.e., the corresponding monodromy matrix has no eigenvalues on the unit circle).

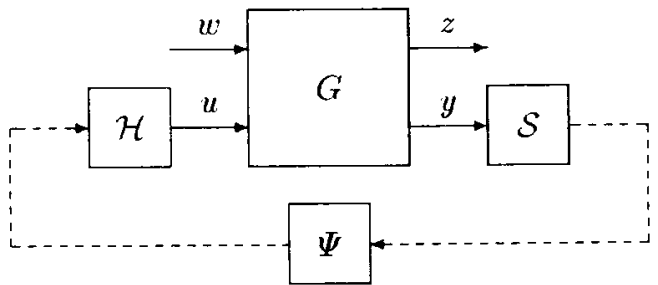

Fig. 3. Sampled-data system with the generalized plant.

Now, let us introduce the following Fourier series expansions:

$$
\begin{aligned}
& B_{\Delta}(t)=\sum_{m=-\infty}^{\infty} B_{\Delta m} e^{j m \omega_{s} t}, \quad C_{\Delta}(t)=\sum_{m=-\infty}^{\infty} C_{\Delta m} e^{j m \omega_{s} t} \\
& D_{\Delta}(t)=\sum_{m=-\infty}^{\infty} D_{\Delta m} e^{j m \omega_{s} t} .
\end{aligned}
$$

Next, let us define the infinite-dimensional Toeplitz matrices by

$$
\begin{aligned}
& \underline{A}_{\Delta}:=\operatorname{diag}\left[\begin{array}{lllll}
\cdots & A_{\Delta} & A_{\Delta} & A_{\Delta} & \cdots
\end{array}\right] \\
& \underline{B}_{\Delta}:=\left[\begin{array}{ccccc}
\ddots & \ddots & \ddots & & \\
\ddots & B_{\Delta 0} & B_{\Delta-1} & B_{\Delta-2} & \\
\ddots & B_{\Delta 1} & B_{\Delta 0} & B_{\Delta-1} & \ddots \\
& B_{\Delta 2} & B_{\Delta 1} & B_{\Delta 0} & \ddots \\
& & \ddots & \ddots & \ddots
\end{array}\right]
\end{aligned}
$$

(similarly for $\underline{C}_{\Delta}$ and $\underline{D}_{\Delta}$ ). Then, the steady-state transfer characteristics of $\Delta$ can be described by

$$
\begin{aligned}
& \underline{\Delta}(j \varphi)=\underline{C}_{\Delta}\left(\underline{E}(j \varphi)-\underline{A}_{\Delta}\right)^{-1} \underline{B}_{\Delta}+\underline{D}_{\Delta} \\
& \underline{E}(j \varphi)=\operatorname{diag}\left[\cdots \quad j \varphi_{-1} I \quad j \varphi_{0} I \quad j \varphi_{1} I \quad \cdots\right] .
\end{aligned}
$$

It is easy to show that $\Delta(j \varphi)$ also defines a bounded operator on $l_{2}$ for each $\varphi \in \mathcal{I}_{0}$.

Definition 3: The $L_{\infty}$-norm of $\hat{\Delta}$ is defined as $\|\hat{\Delta}\|_{\infty}:=$ $\max _{\varphi \in \mathcal{I}_{0}}\|\underline{\Delta}(j \varphi)\|$. In particular, if $\Delta$ is bounded on $L_{2}$, it is also called the $H_{\infty}$-norm of $\hat{\Delta}$.

It is easy to see that if $\Delta$ is FDLTI, the above definitions reduce to the standard ones for the $L_{\infty}$-norm and $H_{\infty}$-norm of $\hat{\Delta}$. We can also show that if $\Delta$ is bounded on $L_{2}$, the $H_{\infty}$-norm of $\hat{\Delta}$ and the $L_{2}$-induced norm of $\Delta$ coincide.

\section{Determinant of a Linear Operator on $l_{2}$}

As mentioned before, we aim to develop a Nyquist-type of argument in this paper. To this end, the notion of the determinant of a "return difference FR-operator" seems indispensable. Although such a notion is not necessarily standard for general linear operators, the following results of [4] and [12] suffice for our purpose (a more elementary treatment is given in [14]).

Consider the operator $I-T$, where $T$ is a finite-rank operator. Then, $T$ has a finite number (in fact, no larger than its rank) of nonzero distinct eigenvalues. The set of such eigenvalues is denoted by $\sigma(T)$. For each $\lambda \in \sigma(T)$, there exists an integer $k_{\lambda}$ such that $\operatorname{ker}(T-\lambda I)^{k}=\operatorname{ker}(T-\lambda I)^{k} \lambda\left({ }^{\forall} k>k_{\lambda}\right)$, and the algebraic multiplicity $\mu_{\lambda}:=\operatorname{dim}\left(\operatorname{ker}(T-\lambda I)^{k}\right)$ is finite.

Definition 4: The determinant of the operator $I-T$, which we denote by $\mathcal{D}(I, T)$, is defined as

$$
\mathcal{D}(I, T):=\prod_{\lambda_{i} \in \sigma(T)}\left(1-\lambda_{i}\right)^{\mu_{\lambda_{i}}}
$$

where $\sigma(T)$ denotes the set of distinct nonzero eigenvalues of $T$. 


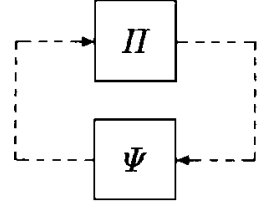

Fig. 4. Discrete-time equivalent $\Sigma_{d}$ of $\Sigma_{s}$.

Remark 1: If $T$ is an operator on a finite-dimensional space, we have $\mathcal{D}(I, T)=\operatorname{det}(I-T)$.

The following result plays an important role.

Proposition 1: If either $S$ or $T$ is finite-rank, then $\mathcal{D}(I, S T)=$ $\mathcal{D}(I, T S)$.

\section{Necessary and Sufficient Condition for Robust Stability}

In this section, we derive a necessary and sufficient condition for robust stability of $\Sigma_{s}$ through a Nyquist-type of argument. Let us consider the discrete-time system $\Sigma_{d}$ shown in Fig. 4, where $\Pi$ is defined by $\Pi:=\mathcal{S} F P \mathcal{H}$. Note that $\Pi$ is LTI even though $P$ may not be. Here, we have the following result, which basically follows from [21, Proposition 7].

Proposition 2: $\Sigma_{s}$ is internally stable if and only if $\Sigma_{d}$ is.

Now, internal stability of $\Sigma_{d}$ can be checked by the discrete-time Nyquist stability criterion, and under the assumption

A0) $\Psi$ internally stabilizes $P_{0}$;

we obtain the following result by Assumption A4) (with any kind of possible unstable cancellations that might occur for some $\Delta \in \boldsymbol{\Delta}$ taken into account [14]).

Proposition 3: Under Assumption A0), $\Sigma_{s}$ is uniformly robustly internally stable if and only if

$$
\inf _{\varphi \in \mathcal{I}_{0}, P \in \mathcal{P}}\left|\operatorname{det}\left(I-\hat{\Pi}\left(e^{j \varphi h}\right) \hat{\Psi}\left(e^{j \varphi h}\right)\right)\right|>0 .
$$

Next, let us relate condition (14) to $G_{\Psi}(j \varphi)$ and $\Delta(j \varphi)$ introduced in Section III. To this end, note that $\hat{\Pi}=\hat{\Pi}_{0}+\hat{\Phi}_{\Delta}$ from (2), where $\Phi_{\Delta}=\mathcal{S} G_{21} \Delta G_{12} \mathcal{H}$ (note that $\mathcal{S} G_{21} \Delta G_{12} \mathcal{H}$ is LTI). Then, it is routine to show that, under $\mathrm{A} 0),(14)$ is equivalent to

$$
\inf _{\varphi \in \mathcal{I}_{0}, \Delta \in \Delta}\left|\operatorname{det}\left(I-\hat{\Lambda}\left(e^{j \varphi h}\right) \hat{\Phi}_{\Delta}\left(e^{j \varphi h}\right)\right)\right|>0 .
$$

Here, we have

$$
\operatorname{det}\left(I-\hat{\Lambda}\left(e^{j \varphi h}\right) \hat{\Phi}_{\Delta}\left(e^{j \varphi h}\right)\right)=\mathcal{D}\left(I, \Delta(j \varphi) \underline{G}_{\Psi}(j \varphi)\right) .
$$

To see this, observe

$$
\begin{aligned}
\mathcal{D} & \left(I, \underline{\Delta}(j \varphi) \underline{G}_{\Psi}(j \varphi)\right) \\
& =\mathcal{D}\left(I, \hat{\Lambda}\left(e^{j \varphi h}\right) \cdot \frac{1}{h} \underline{G_{21}}(j \varphi) \underline{\Delta}(j \varphi) \underline{G_{12} H}(j \varphi)\right)
\end{aligned}
$$

by Proposition 1 and (3). By the impulse modulation formula for $h$ periodic systems [see (36) in Appendix A], (16) follows readily since $\hat{\Lambda} \hat{\Phi}_{\Delta}$ is a finite-dimensional matrix.

To summarize the above, we obtain the following robust stability condition:

$$
\inf _{\varphi \in \mathcal{I}_{0}, \Delta \in \Delta}\left|\mathcal{D}\left(I, \Delta(j \varphi) \underline{G}_{\Psi}(j \varphi)\right)\right|>0 .
$$

By restating the above condition, we can obtain the following main result of this section.

Theorem 1: Under Assumption A0), the sampled-data system $\Sigma_{s}$ is uniformly robustly internally stable if and only if

$$
\left\|\mathcal{G}_{\Psi}\right\|_{\infty}=\max _{\varphi \in \mathcal{I}_{0}}\left\|\underline{G}_{\Psi}(j \varphi)\right\|<1 .
$$

Remark 2: Since $\left\|\mathcal{G}_{\Psi}\right\|_{\infty}$ coincides with the $L_{2}$-induced norm from $w$ to $z$ of the sampled-data system shown in Fig. 3 (as mentioned in Section III-A), (19) is nothing but the small-gain condition in terms of the $L_{2}$-induced norm. What is important here is that the condition has been derived by taking into account unstable perturbations. A similar condition stated in [25, Th. 5.1] was derived by considering only stable perturbations. Also note that, unlike in [25], our perturbation set $\boldsymbol{\Delta}$ does not contain, for example, any sampled-data systems, which are not natural as perturbations for a continuous-time plant.

Proof-i) Sufficiency: The proof is fairly standard, and the details are omitted.

ii) Necessity: Suppose $\left\|\mathcal{G}_{\Psi}\right\|_{\infty} \geq 1$, i.e., $\left\|G_{\Psi}(j \varphi)\right\| \geq 1$ for some $\varphi \in \mathcal{I}_{0}$. Then, for any $\varepsilon_{1}>0$, there exists $\underline{x}, \underline{y}$, and $\gamma$ such that

$$
\gamma \underline{y}=\underline{G}_{\Psi}(j \varphi) \underline{x}, \quad\|\underline{x}\|=\|\underline{y}\|=1, \gamma>1-\varepsilon_{1} .
$$

Let us define $\underline{\Delta}_{1}:=(1 / \gamma) \underline{x} \underline{y}^{*}$. Then, it readily follows that $\underline{\Delta}_{1} \underline{G}_{\Psi}(j \varphi) \underline{x}=\underline{x}$. Also, we have $\left\|\underline{\Delta}_{1}\right\|=1 / \gamma<1 /\left(1-\varepsilon_{1}\right)$. This implies that for any $\varepsilon_{2}>0$, there exists $\Delta_{1}$ of the form $\underline{x}_{1} \underline{y}_{1}^{*}$ such that $\left\|\underline{\Delta}_{1}\right\|<1+\varepsilon_{2}$ and

$$
\mathcal{D}\left(I, \Delta_{1} \underline{G}_{\Psi}(j \varphi)\right)=0 .
$$

By Lemma B2 in Appendix B, there exists $\Delta_{2} \in \boldsymbol{\Delta}$ such that $\left\|\Delta_{1} /\left(1+\varepsilon_{2}\right)-\underline{\Delta}_{2}(j \varphi)\right\|<\varepsilon_{2}$. Thus, it follows that

$$
\begin{aligned}
& \left\|\Delta_{1}-\underline{\Delta}_{2}(j \varphi)\right\| \\
& \quad \leq\left\|\Delta_{1}-\Delta_{1} /\left(1+\varepsilon_{2}\right)\right\|+\left\|\Delta_{1} /\left(1+\varepsilon_{2}\right)-\underline{\Delta}_{2}(j \varphi)\right\| \\
& \quad<2 \varepsilon_{2} .
\end{aligned}
$$

This together with (21) implies that (18) fails.

Q.E.D.

In [17], the present authors and their colleague directly dealt with (19) to derive an equivalent discrete-time $H_{\infty}$ condition. For other earlier arguments which studied the equivalent $L_{2}$-induced norm condition, see, e.g., [3], [20], [23], and [26].

\section{Robust Stability Against LTI Perturbations}

In the preceding section, we studied the "general" case where the perturbation $\Delta$ is linear $h$-periodic. Since the nominal plant is LTI, however, it would be more reasonable to assume that $\Delta$ is also LTI [8], [9], [24]. Hence, in this section, we derive a necessary and sufficient condition for robust stability against LTI (actually FDLTI) perturbations. Also, we compare the obtained condition with the small-gain condition (19) and clarify when the latter becomes particularly poor.

We denote the subset of $\boldsymbol{\Delta}$ consisting of LTI perturbations by $\boldsymbol{\Delta}_{\text {LTI }}$. It is obvious that the uniform robust internal stability condition is given by (18) with $\boldsymbol{\Delta}$ replaced by $\boldsymbol{\Delta}_{\mathrm{LTI}}$

$$
\inf _{\varphi \in \mathcal{I}_{0}, \Delta \in \Delta_{\mathrm{LTI}}}\left|\mathcal{D}\left(I, \Delta(j \varphi) \underline{G}_{\Psi}(j \varphi)\right)\right|>0 .
$$

Here, for $\Delta \in \Delta_{\mathrm{LTI}}$, we have

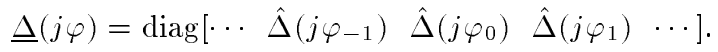

As is clear from the above structure of $\Delta(j \varphi)$, the necessary and sufficient condition (23) turns out to be a highly structured $\mu$-type of condition, as pointed out in [9] (for the case of stable perturbations).

\section{A. Robust Stability Condition Against LTI Perturbations}

Using Proposition 1, together with (3) and (24), let us rewrite (23) as

$$
\inf _{\varphi \in \mathcal{I}_{0}, \Delta \in \Delta_{\mathrm{LTI}}}\left|\operatorname{det}\left(I-\hat{\Upsilon}_{\Delta}\left(e^{j \varphi h}\right)\right)\right|>0
$$


where

$$
\begin{aligned}
\hat{\Upsilon}_{\Delta}\left(e^{j \varphi h}\right):= & \frac{1}{h} \sum_{m=-\infty}^{\infty} \hat{\Lambda}\left(e^{j \varphi h}\right) \hat{G}_{21}\left(j \varphi_{m}\right) \hat{\Delta}\left(j \varphi_{m}\right) \\
& \cdot \hat{G}_{12}\left(j \varphi_{m}\right) \hat{H}\left(j \varphi_{m}\right) .
\end{aligned}
$$

In view of (25), let us fix $\varphi \in \mathcal{I}_{0}$ and consider the set of the series $\left\{\hat{\Delta}\left(j \varphi_{m}\right)\right\}$ in $m$ satisfying $\operatorname{det}\left(I-\hat{\Upsilon}_{\Delta}\left(e^{j \varphi h}\right)\right)=0$, which we denote by $\hat{\boldsymbol{\Delta}}_{\varphi}$ (for $\varphi=0$, we consider only those series such that $\hat{\Delta}\left(j \varphi_{0}\right)$ is real and $\hat{\Delta}\left(j \varphi_{-m}\right)$ is the complex conjugate of $\hat{\Delta}\left(j \varphi_{m}\right)$, similarly for $\left.\varphi=\omega_{s} / 2\right)$. Then, we define

$$
\chi(\varphi):=\inf _{\left\{\Delta\left(j \varphi_{m}\right)\right\} \in \Delta_{\varphi}}\left(\sup _{m}\left\|\hat{\Delta}\left(j \varphi_{m}\right)\right\|\right), \mu(\varphi):=\chi(\varphi)^{-1} .
$$

Now, we can obtain the following theorem.

Theorem 2: Under Assumption A0), the sampled-data system $\Sigma_{s}$ is uniformly robustly internally stable against the LTI perturbations $\boldsymbol{\Delta}_{\text {LTI }}$ if and only if

$$
\mu:=\max _{\varphi \in \mathcal{I}_{0}} \mu(\varphi)<1 .
$$

Proof: The sufficiency part is easy to prove. The proof of the necessity part is done by contradiction. Suppose that $\mu \geq 1$. Then, there exists some $\varphi \in \mathcal{I}_{0}$ such that for any $\varepsilon_{1}>0$, there exists a series $\left\{\hat{\Delta}_{1}\left(j \varphi_{m}\right)\right\} \in \hat{\boldsymbol{\Delta}}_{\varphi}$ satisfying $\left\|\hat{\Delta}_{1}\left(j \varphi_{m}\right)\right\|<$ $1+\varepsilon_{1}\left({ }^{\forall} m\right)$. Therefore, by Lemma B1 in Appendix B, there exists a sequence $\left\{\Delta_{2 k}\right\}$ of systems in $\Delta_{\text {LTI }}$ such that $\| \hat{\Delta}_{1}\left(j \varphi_{m}\right) /(1+$ $\left.\varepsilon_{1}\right)-\hat{\Delta}_{2 k}\left(j \varphi_{m}\right) \|<\varepsilon_{1}(m=0, \pm 1, \cdots, \pm k)$. It follows that

$$
\begin{aligned}
\left\|\hat{\Delta}_{1}\left(j \varphi_{m}\right)-\hat{\Delta}_{2 k}\left(j \varphi_{m}\right)\right\| \leq & \left\|\hat{\Delta}_{1}\left(j \varphi_{m}\right)-\hat{\Delta}_{1}\left(j \varphi_{m}\right) /\left(1+\varepsilon_{1}\right)\right\| \\
& +\left\|\hat{\Delta}_{1}\left(j \varphi_{m}\right) /\left(1+\varepsilon_{1}\right)-\hat{\Delta}_{2 k}\left(j \varphi_{m}\right)\right\| \\
< & 2 \varepsilon_{1} \quad(m=0, \pm 1, \cdots, \pm k) . \quad(29)
\end{aligned}
$$

Therefore, for any $\varepsilon(>0)$, we have $\left|\operatorname{det}\left(I-\hat{\Upsilon}_{\Delta_{2 k}}\left(e^{j \varphi h}\right)\right)\right|<\varepsilon$ for small enough $\varepsilon_{1}$ and large enough $k$ by A6)-A8). This means that (25) fails.

Q.E.D.

In special cases, $\underline{G}_{\Psi}(j \varphi)$ becomes a rank-one matrix so that the set $\hat{\boldsymbol{\Delta}}_{\varphi}$ becomes convex and $\mu(\varphi)$ can be computed analytically.

Theorem 3: Suppose that the plant is a single-input system. Then $\mu(\varphi)$ is attained and is given by

$$
\mu(\varphi)=\sum_{m=-\infty}^{\infty}\left\|\hat{\Lambda}\left(e^{j \varphi h}\right) \hat{G}_{21}\left(j \varphi_{m}\right)\right\|\left\|(1 / h) \hat{G}_{12}\left(j \varphi_{m}\right) \hat{H}\left(j \varphi_{m}\right)\right\| .
$$

Theorem 4: Suppose that the plant is a single-output system. Then $\mu(\varphi)$ is attained and is given by

$$
\mu(\varphi)=\sum_{m=-\infty}^{\infty}\left\|\hat{G}_{21}\left(j \varphi_{m}\right)\right\|\left\|(1 / h) \hat{G}_{12}\left(j \varphi_{m}\right) \hat{H}\left(j \varphi_{m}\right) \hat{\Lambda}\left(e^{j \varphi h}\right)\right\| .
$$

It follows from Theorems $2-4$ that if the plant is either singleinput or single-output, we can readily check robust stability of the sampled-data system $\Sigma_{s}$, given $\Psi$. In particular, if the plant is single-input/single-output, then (30) and (31) coincide and the robust stability condition (28) reduces to

$$
\max _{\varphi \in \mathcal{I}_{0}}\left\|\hat{\Lambda}\left(e^{j \varphi h}\right)\right\| \cdot \eta\left(e^{j \varphi h}\right)<1
$$

where $\eta\left(e^{j \varphi h}\right)$ is a real-valued nonnegative function defined on the unit circle

$$
\eta\left(e^{j \varphi h}\right):=\sum_{m=-\infty}^{\infty}\left\|\hat{G}_{21}\left(j \varphi_{m}\right)\right\|\left\|(1 / h) \hat{G}_{12}\left(j \varphi_{m}\right) \hat{H}\left(j \varphi_{m}\right)\right\| .
$$

Note that $\eta\left(e^{j \varphi h}\right)=\eta\left(e^{-j \varphi h}\right)$ and that the above infinite sum converges for each $\varphi \in \mathcal{I}_{0}$ and defines a continuous function with respect to $\varphi$ by A6)-A8). Therefore, $\eta\left(e^{j \varphi h}\right)$ can be approximated by $\left|\hat{\theta}\left(e^{j \varphi h}\right)\right|$ with a discrete-time stable transfer function $\hat{\theta}(z)$. Since $\hat{\Lambda}(z)$ is a closed-loop discrete-time transfer function, we can (approximately) solve an even harder problem of robust stabilization against LTI perturbations [i.e., the problem of finding $\Psi$ that achieves (32)] by solving a discrete-time $H_{\infty}$ problem with the weight $\hat{\theta}(z)$. Actually, this method is nothing but the one proposed by the present authors in their previous study on robust stabilization of (multirate) sampled-data systems [1], where the condition (32) was regarded as only sufficient.

\section{B. When Does the Small-Gain Condition Become a Poor Measure?}

It was demonstrated in [8] and [9] by an example that the smallgain condition could become extremely poor as a measure for robust stability analysis against LTI perturbations. In order to be more quantitatively specific, and also to get some insight into the synthesis problem of robustly stabilizing controller, let us compare $\left\|\underline{G}_{\Psi}(j \varphi)\right\|$ with $\mu(\varphi)$ and clarify when the former becomes much larger than the latter (i.e., when the small-gain condition becomes particularly inappropriate).

Suppose for simplicity that the plant is single-input. Then, $\underline{G}_{\Psi}(j \varphi)$ becomes a rank-one matrix so that its norm can be computed easily

$$
\begin{aligned}
\left\|\underline{G}_{\Psi}(j \varphi)\right\|= & \left(\sum_{m=-\infty}^{\infty}\left\|\hat{\Lambda}\left(e^{j \varphi h}\right) \hat{G}_{21}\left(j \varphi_{m}\right)\right\|^{2}\right)^{1 / 2} \\
& \cdot\left(\sum_{m=-\infty}^{\infty}\left\|(1 / h) \hat{G}_{12}\left(j \varphi_{m}\right) \hat{H}\left(j \varphi_{m}\right)\right\|^{2}\right)^{1 / 2} .
\end{aligned}
$$

Comparing this with (30), we naturally have $\left\|\underline{G}_{\Psi}(j \varphi)\right\| \geq \mu(\varphi)$ by the Schwarz inequality, where the equality holds if and only if the alignment condition

$$
\left\|\hat{\Lambda}\left(e^{j \varphi h}\right) \hat{G}_{21}\left(j \varphi_{m}\right)\right\|=c_{1}\left\|(1 / h) \hat{G}_{12}\left(j \varphi_{m}\right) \hat{H}\left(j \varphi_{m}\right)\right\| \quad\left({ }^{\forall} m\right)
$$

is satisfied for some constant $c_{1}$. It is similar for the single-output case.

We can interpret the above arguments as follows. Namely, in view of the alignment condition, the small-gain condition could become very poor if the ratio of $\left\|\hat{\Lambda}\left(e^{j \varphi h}\right) \hat{G}_{21}\left(j \varphi_{m}\right)\right\|$ to $\left\|(1 / h) \hat{G}_{12}\left(j \varphi_{m}\right) \hat{H}\left(j \varphi_{m}\right)\right\|$ varies remarkably with those $m$ that correspond to the significant terms in the summations. The example in [8] and [9] can be explained by this observation, where it was shown that the small-gain condition could become extremely poor by changing the bandwidth of the filter $F$ [note that $\hat{F}$ affects $\hat{G}_{21}\left(j \varphi_{m}\right)$ but not $\hat{G}_{12}\left(j \varphi_{m}\right)$ in (35)].

Now, let us consider the following rule of thumb about the choice of the filter $F$ : take the bandwidth of $\hat{F}$ to be equal to $\omega_{s} / 2$ (which follows from the sampling theorem). For the typical case where $\mathcal{H}$ is the zero-order hold, the bandwidth of $\hat{H}$ is also about $\omega_{s} / 2$. Hence, under these conventional choices, the above observation suggests that there is not enough reason to believe that the small-gain condition is an overly poor measure. To put it reversely, as long as we follow the rule of thumb, there seems to be a moderate reason to adhere to the small-gain condition (19) [because it may not pay to design a controller based on the exact but much involved condition (32)].

\section{CONCLUSION}

In this paper, we gave necessary and sufficient conditions for robust stability of sampled-data systems under possibly unstable 


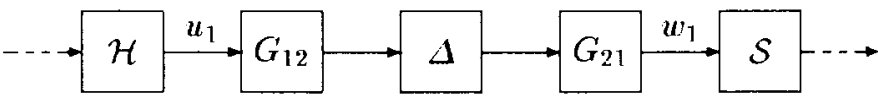

Fig. 5. Sampled-data system including an $h$-periodic continuous-time system.

additive/multiplicative perturbations. The results were established by a Nyquist-type of argument applied to the frequency-domain method of sampled-data systems developed recently in [2] and [28]-[30], and the robust stability problems for linear $h$-periodic perturbations and LTI perturbations were dealt with in a unified manner. Furthermore, the relationship between the two problems was clarified, and some insight into the design problem of a robustly stabilizing controller was obtained.

\section{APPENDIX A}

\section{IMPUlSe MOdUlation FORMUla FOR $h$-PERIDOIC SySTEMS}

Consider the open-loop sampled-data system shown in Fig. 5, where $\Delta$ is linear $h$-periodic and $G_{12}$ and $G_{21}$ are LTI and satisfy A7). We further assume A8). Let us define the LTI discrete-time system $\Phi_{\Delta}:=\mathcal{S} G_{21} \Delta G_{12} \mathcal{H}$. Our purpose here is to relate $\hat{\Phi}_{\Delta}\left(e^{j \varphi h}\right)$ to $\hat{H}\left(j \varphi_{m}\right), \hat{G}_{12}\left(j \varphi_{m}\right), \hat{G}_{21}\left(j \varphi_{m}\right)$, and $\Delta(j \varphi)$.

We can show the relation

$$
\hat{\Phi}_{\Delta}\left(e^{j \varphi h}\right)=\frac{1}{h} \underline{G_{21}}(j \varphi) \triangleq(j \varphi) \underline{G_{12} H}(j \varphi)
$$

which we call the impulse modulation formula for $h$-periodic systems (the proof can be found in [14]). If $\Delta$ is LTI, by (24) it reduces to the well-known formula for LTI systems [22]

$$
\hat{\Phi}_{\Delta}\left(e^{j \varphi h}\right)=\frac{1}{h} \sum_{m=-\infty}^{\infty} \hat{G}_{21}\left(j \varphi_{m}\right) \hat{\Delta}\left(j \varphi_{m}\right) \hat{G}_{12}\left(j \varphi_{m}\right) \hat{H}\left(j \varphi_{m}\right) .
$$

In the above, we assumed that $G_{21} \Delta G_{12}$ is strictly causal. If this is not the case, $w_{1}$ may be discontinuous at sampling instants so that half of the direct feedthrough matrix of $G_{21} \Delta G_{12} H$ evaluated as the limit from the right of $t=0$ must be added to the right-hand side of (36) and (37) in that case.

\section{APPENDIX B}

\section{SOME RESUlTS ON APPROXIMATE INTERPOLATION}

In this Appendix, we give some results on approximate interpolation of given frequency response data by the frequency-domain representation of a finite-dimensional system. The proofs can be found in [14] and [16].

Lemma B1: Suppose that any $\varphi \in \mathcal{I}_{0}$ and complex matrices (of the same size) $D_{m}$ with $\left\|D_{m}\right\|<1(m=0, \pm 1, \cdots, \pm k)$ are given (for $\varphi=0$, we assume that $D_{0}$ is real and $D_{-m}$ is the complex conjugate of $D_{m}$, similarly for $\varphi=\omega_{s} / 2$ ). Then, for any $\varepsilon>0$, there exists a stable causal FDLTI system $\Delta$ such that

$$
\begin{aligned}
\|\hat{\Delta}\|_{\infty} & <1,\left\|D_{m}-\hat{\Delta}\left(j \varphi_{m}\right)\right\|<\varepsilon \quad(m=0, \pm 1, \cdots, \pm k) \\
\left\|\hat{\Delta}\left(j \varphi_{m}\right)\right\| & <\varepsilon \quad(|m|>k) .
\end{aligned}
$$

Lemma B2: Suppose that any $\varphi \in \mathcal{I}_{0}$ and $\Delta=\underline{x} \underline{y}^{*}$ such that $\|\underline{\Delta}\|<1$ are given, where $\underline{x}$ and $y$ are infinite-dimensional vectors. Then, for any $\varepsilon>0$, there exists a stable $h$-periodic system $\Delta_{1}$ described by (9) such that

$$
\left\|\hat{\Delta}_{1}\right\|_{\infty}<1, \quad\left\|\underline{\Delta}-\Delta_{1}(j \varphi)\right\|<\varepsilon .
$$

\section{ACKNOWLEDGMENT}

T. Hagiwara is grateful to Prof. B. A. Francis for valuable discussions and comments on this work.

\section{REFERENCES}

[1] M. Araki and T. Hagiwara, "Robust stability of sampled data systems," in Robust Control, S. Hosoe, Ed. New York: Springer, 1992.

[2] M. Araki, Y. Ito, and T. Hagiwara, "Frequency response of sampled-data systems," Automatica, vol. 32, no. 4, pp. 483-497, 1996.

[3] B. A. Bamieh and J. B. Pearson, "A general framework for linear periodic systems with applications to $H_{\infty}$ sampled-data control," IEEE Trans. Automat. Contr., vol. 37, pp. 418-435, Apr. 1992.

[4] A. Böttcher and B. Silbermann, Analysis of Toeplitz Operators. Berlin, Germany: Springer, 1990.

[5] M. J. Chen and C. A. Desoer, "Necessary and sufficient condition for robust stability of linear distributed feedback systems," Int. J. Contr., vol. 35 , no. 2, pp. 255-267, 1982.

[6] T. Chen and B. A. Francis, "Input-output stability of sampled-data control systems," IEEE Trans. Automat. Contr., vol. 36, pp. 50-58, Jan. 1991.

[7] _ , "On the $L_{2}$-induced norm of a sampled-data system," Syst. Contr. Lett., vol. 15, pp. 211-219, 1990.

[8] G. Dullerud and K. Glover, "Necessary and sufficient conditions for robust stability of SISO sampled-data systems to LTI perturbations," in Proc. Amer. Control Conf., 1992, pp. 2644-2648.

[9] _ _ "Robust stabilization of sampled-data systems to structured LTI perturbations," IEEE Trans. Automat. Contr., vol. 38, pp. 1497-1508, Oct. 1993.

[10] _ , "Analysis of structured LTI uncertainty in sampled-data systems," Automatica, vol. 31, no. 1, pp. 99-113, 1995.

[11] J. S. Freudenberg, R. H. Middleton, and J. H. Braslavsly, "Inherent design limitations for linear sampled-data feedback systems," Int. J. Contr., vol. 61, no. 6, pp. 1387-1421, 1995.

[12] I. C. Gohberg and M. G. Krein, Introduction to the Theory of Linear Nonselfadjoint Operators. American Math. Soc., 1969.

[13] G. C. Goodwin and M. Salgado, "Frequency domain sensitivity functions for continuous time systems under sampled data control," Automatica, vol. 30, no. 8, pp. 1263-1270, 1994

[14] T. Hagiwara and M. Araki, "Robust stability of sampled-data systems under possibly unstable additive/multiplicative perturbations," Dept. Electrical Engineering, Kyoto Univ., Tech. Rep. no. 94-10, 1994.

[15] _ , "FR-operator approach to the $\mathrm{H}_{2}$ analysis and synthesis of sampled-data systems," IEEE Trans. Automat. Contr., vol. 40, pp. 1411-1421, Aug. 1995.

[16] _ _ "Robust stability conditions of sampled-data systems: A Nyquisttype approach with FR-operators," in Proc. 18th SICE Symp. Dynamical System Theory, 1995, pp. 171-176.

[17] T. Hagiwara, Y. Ito, and M. Araki, "Computation of the frequency response gains and $H_{\infty}$-norm of a sampled-data system," Syst. Contr. Lett., vol. 25, no. 4, pp. 281-288, 1995.

[18] S. R. Hall and N. M. Wereley, "Generalized Nyquist stability criterion for linear time periodic systems," in Proc. Amer. Control Conf., 1990, pp. $1518-1525$.

[19] S. Hara, M. Nakajima, and P. T. Kabamba, "Robust stabilization in digital control systems," in Recent Advances in Mathematical Theory of Systems, Control, Networks and Signal Processing I. Tokyo: Mita, 1992.

[20] Y. Hayakawa, S. Hara, and Y. Yamamoto, " $H_{\infty}$ type problem for sampled-data control systems-A solution via minimum energy characterization," IEEE Trans. Automat. Contr., vol. 39, pp. 2278-2284, Nov. 1994.

[21] P. A. Iglesias, "Input-output stability of sampled-data linear timevarying systems," IEEE Trans. Automat. Contr., vol. 40, pp. 1646-1650, Sept. 1995.

[22] E. I. Jury, Sampled-Data Control Systems. New York: Wiley, 1958

[23] P. T. Kabamba and S. Hara, "Worst-case analysis and design of sampled-data control systems," IEEE Trans. Automat. Contr., vol. 38, pp. 1337-1357, Sept. 1993.

[24] Y. Oishi, "Computation-oriented expression of a nonconservative condition for robust stability of sampled-data systems," Int. J. Contr., vol. 62, no. 5, pp. 1085-1104, 1995.

[25] N. Sivashankar and P. P. Khargonekar, "Robust stability and performance analysis of sampled-data systems," IEEE Trans. Automat. Contr., vol. 38, no. 1, pp. 58-69, 1993. 
[26] H. T. Toivonen, "Sampled-data control of continuous-time systems with an $H_{\infty}$ optimality criterion," Automatica, vol. 28 , no. 1 , pp. 45-54, 1992.

[27] N. M. Wereley and S. R. Hall, "Frequency response of linear time periodic systems," in Proc. Conf. Decision and Control, 1990, pp. 3650-3655.

[28] Y. Yamamoto, "On the state space and frequency domain characterization of $H_{\infty}$-norm of sampled-data systems," Syst. Contr. Lett., vol. 21, pp. 163-172, 1993.

[29] Y. Yamamoto and M. Araki, "Frequency responses for sampled-data systems-Their equivalence and relationships," Linear Algebra and Its Appl., vols. 205/206, pp. 1319-1339, 1994.

[30] Y. Yamamoto and P. P. Khargonekar, "Frequency response of sampleddata systems," IEEE Trans. Automat. Contr., vol. 41, pp. 166-176, Feb. 1996. 